\title{
Determination and Use of the Sag Point as a Reference Point in the Heating of Glasses
}

\author{
Sam Spinner, Given W. Cleek, and Edgar H. Hamilton
}

\begin{abstract}
Glasses, when heated, undergo a gradual continuous change from solids to liquids over a rather wide temperature range. As a consequence, it has been found necessary to define certain temperature reference points in this transition range so that different glasses may be compared with each other. These points are chosen to correspond to established stages in the annealing or forming process. A new reference point, designated as the sag point, is described. The experimental determination of this point is rapid and simple. Also, it gives useful information concerning the annealing temperatures and ease of formation of glasses of widely varying compositions.

The sag point is defined as the temperature at which a glass fiber 0.5 to 0.8 millimeter in diameter, horizontally supported at $1 / 2$-inch intervals, will sag under its own weight in $25 \pm 5$ minutes.
\end{abstract}

\section{General}

One of the more widely accepted definitions of glass is that it is a material that has cooled from the liquid state without undergoing a change in phase [1]. ${ }^{1}$ Though glass may be regarded either as a supercooled liquid or an amorphous solid, the latter designation is more appropriate to stress the fact that ostensibly glass behaves like a solid, i. e., it retains its shape and reacts elastically to shear and tensile stresses. The process of transition from liquid to glass on cooling or from glass to liquid on heating is a continuous one. There is no sharply defined temperature, corresponding to the melting point of a crystal, at which it can be said that glass melts. Glass technologists have found it necessary, therefore, as a practical matter, to define certain reference points in the heating or cooling process so that reasonable comparisons can be made between glasses of different compositions, similar to the comparison of crystals by their melting points. Because the reference points for glass are not sharply defined, their selection is, in a sense, arbitrary. Their choice, however, is governed by established procedures in the annealing, forming, or other stages in the production process.

Glasses of different compositions have different temperature-viscosity relationships. Hence, glasses whose reference points might correspond in one temperature region, such as the annealing range, will, in all probability, not have corresponding points in another region such as the forming range. Considerations of this nature show why more than one reference point is necessary.

Littleton and Roberts [2] and Littleton [3] made the original contributions to the establishment of reference points by defining successively the annealing and softening points. Significant refinements and additions have been made by Lillie [4]. The American Society for Testing Materials [5] defines several such points now in use. These points are usually defined in terms of a specific phenomenon associated with a set of experimental operations, and

${ }^{1}$ Figures in brackets indicate the literature references at the end of this paper also in terms of the viscosity of the glass at the temperature of this phenomenon. For instance, the deformation point is defined as "the temperature observed during the measurement of expansivity by the interferometer method at which viscous flow exactly counteracts thermal expansion. The deformation point generally corresponds to a viscosity in the range from $10^{11}$ to $10^{12}$ poises." The point defined by an operational procedure is preferable because the experimental procedure can usually be reproduced more accurately than the viscosity can either be calculated from the experimental conditions, or measured at the same temperature by some independent method. Furthermore, the viscosity frequently is not the only determining parameter. In the case of the softening point, for instance, the density of the glass must also be considered. The kinematic viscosity, then, would be the relevant factor. The situation is further complicated by the fact that at a lower temperature a considerable time may be required before the glass comes to the equilibrium viscosity for that temperature $[6,7]$. This fact has led Lillie to regard the strain point, which occurs in such a low temperature region, to be of limited significance [4b]. It may also be mentioned, in this connection, that Adams, in his well-known paper on the annealing of glass [8], doubted whether the viscosity, which is designated as a megoscopic property, was really related to the release of stress, which involves only microscopic processes.

In the Bureau's Glass Section laboratory, it became necessary to devise a reference point that would serve as a reliable guide for annealing a large number of glasses of widely varying compositions. In order to be serviceable, it was also necessary for the determination of this point to be rapid and reproducible.

It seems desirable to summarize some of the requirements, which a good reference point should satisfy, as follows:

(1) The apparatus and procedure should be simple so that they may be easily duplicated by other laboratories. Methods that require elaborate specimen preparation, for instance, would be undesirable. In general, the fewer qualifying specifications that need be made, the better. 
(2) The method should yield reproducible results. In order to fulfill this most important requirement, the effect sought for should vary sharply with temperature and, conversely, should be relatively insensitive to extraneous factors. If, for instance, the specimen measured is in the fiber form, the thickness of the fiber should not be critical.

(3) The determination should be rapid. This condition is especially important when the specimen is used as a control test for some portion of the manufacturing process.

(4) The value of such a point is obviously enhanced, if significant additional information is obtained.

A reference point satisfying these requirements was suggested by C. A. Faick, formerly of this Bureau, in 1948 , and has been utilized successfully ever since. This point is hereinafter designated as the sag point. It is about $40^{\circ} \mathrm{C}$ higher than the deformation point, usually regarded as the upper limit of the annealing region. In the technique of determining the sag point, it was found that additional significant information could be gained as to the feasibility of making sizable melts of hitherto untried compositions. This will be discussed later.

It is the purpose of this paper to define the sag point, to describe the experimental equipment and procedure for its determination, and to show some typical results with glasses of different compositions. The sag points are compared with the deformation and softening points.

\section{Experimental Technique}

The technique about to be described for determining the sag point bears some similarity to that originated by Silverman [9] and refined by Grauer and Hamilton [10] for measuring the liquidus temperatures of glasses.

\subsection{Equipment}

The basic equipment consists of a temperature gradient furnace, shown in figure 1 , and a platinum. specimen holder, figure 2 . The platinum holder is 6 in. long with supports for the fibers spaced approximately $1 / 2$ in. apart.

The furnace is essentially the same as that described by Grauer and Hamilton [10] except that the heating coils are of Nichrome rather than platinum wire. Figure 3 shows a typical curve of the temperature distribution along the length of the furnace. The temperature range, as well as the gradient, can be altered considerably by proper adjustment of the power controls.

\subsection{Procedure}

A specimen is drawn in the form of a fiber, 0.5 to $0.8 \mathrm{~mm}$ in diameter. Suitable fibers can be prepared by drawing from the lip of the crucible in which the glass was melted. This is a convenient method of obtaining fibers directly from the melt, even in those cases where the glasses devitrify readily. The

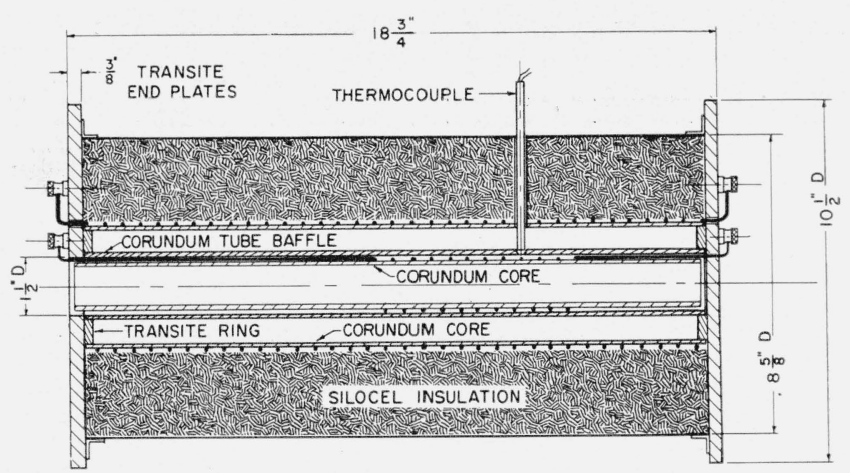

Figure 1. Temperature gradient furnace.

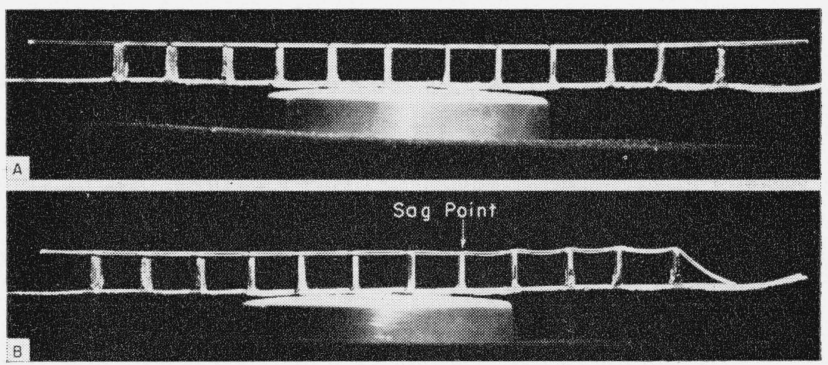

Figure 2. Platinum holder with glass fiber specimen. $A$, Before insertion in furnace; B, after withdrawal from furnace.

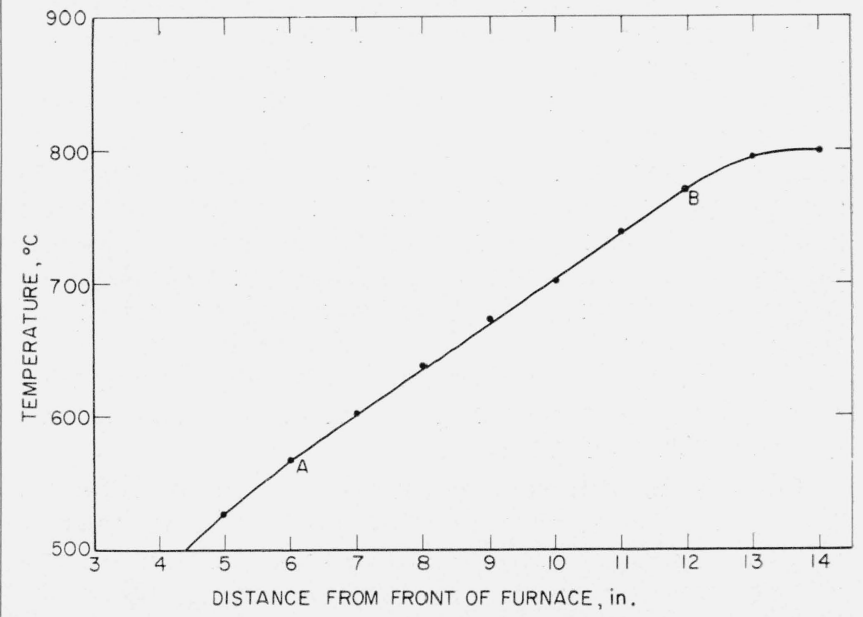

FIGURE 3. Typical temperature distribution curve along length of gradient furnace.

specimen is placed lengthwise on the holder (see fig. 2A) and the holder is inserted into the core of the furnace. After $25 \mathrm{~min}$ the holder is withdrawn. If the furnace has been properly adjusted, that section of the fiber that was in the hotter region will have sagged between the supports, whereas the section of fiber that remained in the cooler region will not have sagged. The temperature at the first compartment, measured from the open end of the furnace, in which the glass sagged between the supports (fig. 2B) is the temperature of the sag point. 
The furnace is properly adjusted when the sag point occurs in the linear gradient region of the temperature distribution curve, shown between points $\mathrm{A}$ and $\mathrm{B}$ in figure 3 . A probe thermocouple is then inserted into the furnace up to this sag point distance and the temperature is measured.

\subsection{Definition}

Following the above procedure, the sag point is defined as the temperature at which a fiber, 0.5 to $0.8 \mathrm{~mm}$ in diameter, horizontally supported at $1 / 2$-in. intervals, will sag under its own weight in $25 \pm 5 \mathrm{~min}$.

\subsection{Precision of Results}

\section{a. General Considerations}

Under the conditions given in figure 3 , repeated measurements with different specimens of the same glasses, but with the position of the holder varied slightly in the furnace, indicated a reproducibility of the order of $\pm 7^{\circ} \mathrm{C}$. This is the limiting precision for the determination of the sag point and follows from the condition that $\frac{1}{2} \mathrm{in}$. of furnace length corresponds to a temperature change of about $15^{\circ} \mathrm{C}$. Increased precision of results may be obtained by decreasing the over-all temperature gradient and hence the gradient per compartment. In this case, the following factors must be given greater consideration: Maintenance of a uniform distance between supports of the holder, the fiber diameter, and measurement of the temperature. It may also be noted here that it was not found necessary to reinsert the platinum holder in the furnace when measuring the temperature of the sag point with the probe thermocouple. Within the experimental precision of the measurement, no difference was observed whether the holder was in or out of the furnace. The apparatus and method described here were found to give the speed, convenience, and precision sufficient for the purpose of the determination.

\section{b. Effect of Fiber Diameter}

As might be expected, the temperature of the sag point was found to increase somewhat with increased fiber diameter. Within the precision used here, no significant variation was found within the given fiber diameter tolerance. The following table for one glass, BSC 517, shows typical data relating fiber diameter to sag point.

\begin{tabular}{|c|c|}
\hline Diameter & Sag point \\
\hline & \\
\hline$m m$ & $\circ C$ \\
0.50 to 0.84 & 650 \\
.52 to .69 & 650 \\
.50 to .60 & 657 \\
.55 to .70 & 657 \\
.62 to .76 & 650 \\
& \\
\hline
\end{tabular}

It may also be noted that the diameter for any single fiber may vary over the specified range without affecting the reproducibility of the result.

\section{c. Effect of Time}

The sag point was found to be relatively insensitive to time in the furnace within rather broad limits. Although longer times may be used safely, less than 20 min may not give reproducible results. The time is not critical within the specified $25 \pm 5 \mathrm{~min}$. One of the conveniences of the method consists in the fact that it is not necessary for the operator to observe the specimen during the time of test, as is usually the case with other reference point determinations [11].

\section{Use of Sag Point in Problems Pertaining to Glass Formation}

As was mentioned earlier, significant information could be obtained in the process of determining the sag point as to the feasibility of cooling sizable melts of new compositions to form glasses. In considering the problem of the glass-forming possibilities of a melt of a new composition, one is interested not only in whether the melt will cool as a glass, but whether glass will form in sufficiently large quantity so that sizable articles can be fabricated from it. Once the glass has formed, one is further concerned if such articles can be annealed without danger of devitrifying. It is necessary to recall that the liquidus temperature is the temperature above which crystals cannot form. The temperature of maximum rate of crystal growth is usually 50 to $100^{\circ} \mathrm{C}$ below the liquidus temperature [10]. This region of maximum crystal growth is of the greatest concern in the practical problem of cooling a melt to glass. As the temperature is decreased below this region, the rate of crystal growth decreases rapidly because the increased viscosity associated with lower temperatures counteracts the tendency toward crystal growth. For practical purposes, this rate becomes. zero at sufficiently low temperatures. Many compositions that will form glasses in small quantities, or when the melt is poured on a cold plate, will devitrify to a considerable extent when an attempt is made to make these same compositions in large quantities. for melts of about $500 \mathrm{~g}$ or more, because the center of the cooling piece must remain at temperatures in this region of maximum crystal growth for considerable periods of time. Thus, when specifying whether a particular composition will form a glass, the experimental conditions under which glass formation occurs must be specified [12].

In the technique of determining the sag point, a portion of the specimen is usually held in the temperature region where crystal growth may occur. The presence of crystals on withdrawal from the furnace, and their relation to the sag point, gives an indication of the tendency of the particular glass to devitrify in this critical region. As a consequence, the feasibility of cooling large melts of a given composition as glass can be predicted.

The one extreme would be a specimen that emerges without the sag point having developed, and yet shows the presence of crystals along the section that 
was in the hotter region of the furnace. Such a composition would present the least favorable condition for glass formation. The opposite, and most favorable condition for glass formation, would be a specimen that emerges with a well-defined sag point and shows no evidence of crystallization anywhere along the fiber.

The fact that crystals do not form in the temperature region above the sag point does not necessarily indicate that the temperature of maximum crystallization is higher than the hottest portion of the specimen. It may indicate that the rate of crystallization is so slow that crystals do not form in the time that the fiber was in the furnace. This shows that the melt can be held for considerable time in this dangerous region with little fear of devitrification.

In general, the difficulty of glass formation increases as crystals appear closer to the sag point. If crystals form on the cold side of the sag point, it indicates that even if glasses are formed, the problem of crystallization during the process of annealing would still remain.

If a glass crystallizes in the annealing temperature range, the difficulties involved are not necessarily insurmountable. Fused silica, for instance, crystallizes in the annealing region, the crystal growth being initiated at the surface. It would appear, however, that if the criterion suggested by this procedure, and by what has been said before, is adopted, then fused silica is far from being the ideal glass it is frequently described to be. If a glass is regarded as the opposite of a crystal, then those glasses that devitrify least readily come closest to being "ideal" from a theoretical as well as a practical point of view. Thus, glasses that contain "network modifiers" and "intermediates" in addition to the so-called basic silica network, but which crystallize fairly slowly in the region of maximum crystal growth, come closer to being "ideal" than does pure silica. On the other hand, pure $\mathrm{B}_{2} \mathrm{O}_{3}$ can be crystallized only with the greatest difficulty and is more nearly an ideal glass in spite of certain (usually) undesirable properties (i. e., high hygroscopicity, low melting point, high coefficient of expansion).

\section{Results}

Table 1 lists several optical glasses ${ }^{2}$ comparing the temperatures of the deformation point, sag point, and softening point. The fiber diameter chosen for the sag point determination $(0.50$ to $0.80 \mathrm{~mm}$ ) is practically the same as that used for the softening point $(0.55$ to $0.75 \mathrm{~mm})$ [14] so that no correction for this dimension is necessary in comparing these two points. The last two columns give successively the difference between the softening point and deformation point, and the difference between the sag point and the deformation point. For the various glasses, these reference points vary, the differences between them vary, and the sag points lie between the deformation and the soitening

\footnotetext{
2 The compositions as well as the meaning of the symbols designating these glasses may be found in reference [13].
}

TABLE 1. Temperatures of reference points of several optical glasses

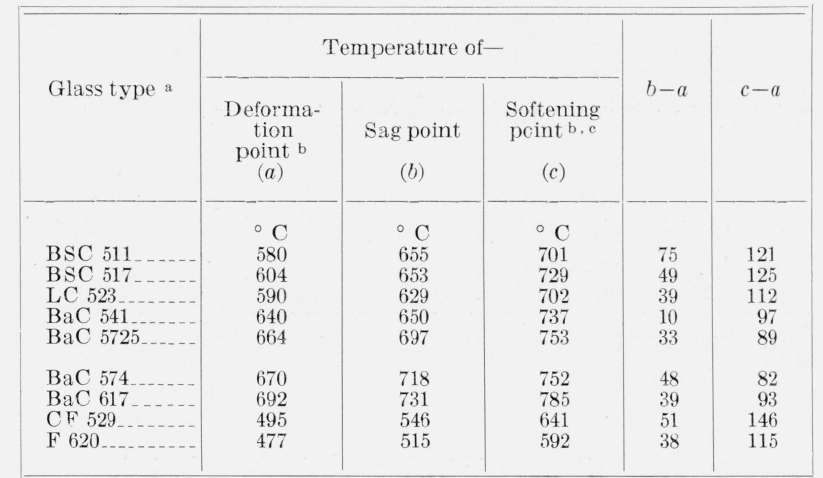

a Compositions are given in [13], Les Shartsis and Sam Spinner, Viscosity and density of molten optical glasses, J. Research VBS 46, 17 o-194 (1951) R P2190. b ASTM Standards, Standard definitions of terms relating to glass and glas products, C162-52 (1955)

c ASTM Standards, Tentative method of test for softening point of glass, C333-54T (1955).

points. This is an example of the fact, mentioned earlier, that the temperature-viscosity relations of glasses of different compositions can vary widely, and consequently of the necessity for using several points to specify more fully the change in properties of different glasses as they are heated. The particular reference point chosen is governed by its ease of determination and proximity to the temperature range of the particular process involved.

The sag point is not offered to replace either the deformation or the softening point. The deformation point is obtained from the determination of the thermal expansion by the interferometric method and is part of the data that are essential to establishing the correct temperature to use for the fine annealing of optical glasses. The sag point is determined in a matter of minutes as compared with a day or more for the deformation point, and can be used to establish a maximum temperature for the comparable annealing of experimental glasses. The temperature of the sag point is nearer to the annealing range than is the softening point and hence devitrification troubles encountered in the latter determination are avoided.

\section{Summary}

(1) A new reference point, the sag point, in the heating (or cooling) of glasses has been defined and the method for experimentally determining this point described. The determination is rapid and simple.

(2) The sag point has proved itself to be of considerable value in determining the maximum annealing temperature of new glasses of widely varying compositions.

(3) A method is also shown for obtaining additional information concerning the devitrification tendencies of experimental melts and the feasibility of cooling them as homogeneous glasses.

(4) Data have been presented in which the sag point is compared to previously defined reference points in the heating of glasses. 


\section{References}

[1] George W. Morey, Properties of glass, Reinhold Publishing Corp., 330 West 42 d St., New York 18, N. Y., 2 d ed., 24-28 (1954)

[2] J. T. Littleton and E. H. Roberts, A method for determining the annealing temperature of glass, J. Opt. Soc. Amer. 4, 224-29 (1920).

[3] (a) J. T. Littleton, A method for measuring the softening temperature of glasses, J. Am. Ceram. Soc. 10, 259-63 (1927); (b) J. T. Littleton, The softening point of glass, J. Soc. Glass Technol. 24, 176 (1940).

[4] (a) H. R. Lillie, Viscosity of glass between the strain point and melting temperature, J. Am. Ceram. Soc. 14, 502-11 (1931); (b) H. R. Lillie, A method for measuring the flow point of glass, J. Am. Ceram. Soc. 35, 149-55 (1952); (c) H. R. Lillie, Re-evaluation of glass viscosities at annealing and strain points, J. Am. Ceram. Soc. 3\%, 111-17 (1954).

[5] Am. Soc. Testing Materials, Standards, Standard definitions of terms relating to glass and glass products, C $162-52$ (1955).

[6] V. H. Stott, E. Irvine, and D. Turner, Viscosity measurements with glass, Proc. Roy. Soc. London $[\mathrm{A}]$ 108, $154(1925)$

[7] H. R. Lillie, Viscosity-time-temperature relations in glass at annealing temperatures, J. Am. Ceram. Soc. 16, 619 (1933).
[8] L. H. Adams, The annealing of glass as a physical problem, J. Franklin Inst. 216, 39-71 (July 1933).

[9] W. B. Silverman, Effect of alumina on devitrification of soda-lime-silica glasses, J. Am. Ceram. Soc. 22, 378 (1939).

[10] O. H. Grauer and E. H. Hamilton, An improved apparatus for the determination of liquidus temperatures and rates of crystal growth in glasses, J. Research NBS 44, 495-502 (1950) RP2096.

[11] L. K. Kowalew and R. L. Shuster, Determination of the softening temperature of glass, Vestnik Akad. Nauk Kazakh. S. S. R. 1, 81-5 (1953); Glastech. Ber. 28, 313 (1955).

[12] Given W. Cleek and Edgar H. Hamilton, Properties of barium titanium silicate glasses, J. Research NBS 37, 317-323 (1956) RP2720.

[13] Leo Shartsis and Sam Spinner, Viscosity and density of molten optical glasses, J. Research NBS 46, 176-194 (1951) RP2190.

[14] Am. Soc. Testing Materials, Standards, Tentative method of test for softening point of glass, C 338-54T (1955).

Washington, February 15, 1957. 